\title{
Synthetic Aperture Flow Imaging Using a Dual Stage Beamformer Approach
}

\author{
Li, Ye; Jensen, Jørgen Arendt
}

Published in:

Proceedings of the 2010 IEEE International Ultrasonics Symposium

Link to article, DOI:

10.1109/ULTSYM.2010.0486

Publication date:

2010

Document Version

Early version, also known as pre-print

Link back to DTU Orbit

Citation (APA):

$\mathrm{Li}$, Y., \& Jensen, J. A. (2010). Synthetic Aperture Flow Imaging Using a Dual Stage Beamformer Approach. In Proceedings of the 2010 IEEE International Ultrasonics Symposium (pp. 1924-1927). IEEE. https://doi.org/10.1109/ULTSYM.2010.0486

\section{General rights}

Copyright and moral rights for the publications made accessible in the public portal are retained by the authors and/or other copyright owners and it is a condition of accessing publications that users recognise and abide by the legal requirements associated with these rights.

- Users may download and print one copy of any publication from the public portal for the purpose of private study or research.

- You may not further distribute the material or use it for any profit-making activity or commercial gain

- You may freely distribute the URL identifying the publication in the public portal

If you believe that this document breaches copyright please contact us providing details, and we will remove access to the work immediately and investigate your claim. 


\title{
Synthetic Aperture Flow Imaging Using a Dual Stage Beamformer Approach
}

\author{
Ye Li and Jørgen Arendt Jensen \\ Center for Fast Ultrasound Imaging, Department of Electrical Engineering, Build. 349 \\ Technical University of Denmark, 2800, Kgs. Lyngby, Denmark
}

\begin{abstract}
A new technique for synthetic aperture flow imaging is presented in this paper. The objective is to increase the frame rate and still maintain a beamforming quality sufficient for flow estimation that is possible to implement in a commercial scanner. A dual stage beamformer is used to beamform the data. In the first stage data are beamformed with a fixed transmit and receive focal point to obtain conventional RF lines. Then the fully dynamic second beamformer uses the data from the first stage to beamform the full image. High resolution image (HRI) lines obtained from the second stage beamformer are input to the velocity estimator. There are four emissions in each emission sequence, which repeat over time. In each emission 32 elements are excited for transmission and 64 elements are used for receiving signals. Thus, four low resolution lines generated in the first stage are input to the second stage beamformer to generate a HRI. Velocities are estimated by cross correlating RF lines $n$ and $n+N$, where $n$ is the global emission index and $N$ is the number of emissions in one sequence. Furthermore, the velocity profile can be obtained by dividing $\mathrm{RF}$ lines into small segments, thus, they are calculated as a function of depth. The performance of this approach has been studied using simulations by Field II and different parameters are studied to reveal the robustness of this approach. For different spacings between emission lines $[2,6,10,14] \lambda$, the standard deviation and the bias of the axial velocity component were $[1.92,2.0,2.07,2.21] \%$ and $[0.61,1.07$, $1.29,1.5] \%$, respectively, compared to the peak velocity. With a spacing of $10 \lambda$, varying the number of emission sets $[1,2,4,6,8]$ used for averaging resulted in standard deviations of [7.14, 2.28, $2.07,1.92,1.36] \%$ and bias $[11.43,2.36,1.29,1.29,1.22] \%$. The parameter study showed that larger spacings gave an increased standard deviation and bias. Furthermore, performance improves if more emissions sets are used for averaging. The performance of the simulation indicates that this dual beamformer approach was able to estimate the flow velocity.
\end{abstract}

\section{INTRODUCTION}

The conventional ultrasound method for acquiring color flow mapping (CFM) has two major limitations: the low frame rate and that the velocity estimates are angle dependent.

With the conventional method, the image consists of many image lines and each of them are obtained by using multiple pulse emissions in that direction. Therefore, the frame rate is low if more image lines are needed. The low frame rate makes the velocity measurement range small and makes it impossible to identify the rapid temporal variations in the blood vessel. The other major limitation is the angle. Only the axial velocity component can be measured in conventional CFM which is a major limitation.

Compared to conventional ultrasound imaging, SA imaging can obtain data continuously and make the frame rate higher and obtain a dynamically focused image in both transmit and receive [1]. Synthetic aperture (SA) techniques have been investigated by several authors [2] [3] [4]. The idea behind SA is to obtain a HRI by adding all the low resolution images (LRI) from the emission sequence. The spherical wave is supposed to be emitted from a single element to cover a large scatterer area. After every emission, a new HRI can be obtained by replacing the latest LRI, thus, yielding continuous HRIs. Those are important issues for flow estimation. In SA imaging a HRI is available at every pulse emission, because of the continuous data acquisition. Therefore, velocities throughout the image can be estimated at the same time. Furthermore, the velocity can be found in any direction, because focusing can be performed in all directions.

However, it is not easy to implement SA on a scanner because of the very high number of calculations for making real-time imaging. Therefore, it is of interest to develop a method that can increase the frame rate and is able to reduce the system implementation complexity at the same time. The dual stage beamformer approach is an alternative to achieve images depth independent focusing without degrading the frame rate and make it possible to implement on a scanner with few calculations.

\section{A. Dual stage beamformer method}

The dual beamformer approach is a two-stage beamforming procedure. In the first stage the data are beamformed using a fixed focus. The focused RF lines are the input to the second beamformer, which is fully dynamically focused. The concept of considering transmit and receive focal points as virtual sources is the basis for calculating the time-of-flight (TOF) [5], [6].

The first stage image lines are obtained by emitting and receiving signals from an active sub-aperture, which moves along the transducer with emission index. Applying the delay profile on the sub-aperture yield focused ultrasound beams. At every emission a single image line is beamformed along the beam direction. The whole image consists of many acquired lines, which are the input to the second beamformer. The receive focal point coincides with the transmit focal point and the round trip TOF is calculated in the second stage beamformer by tracing the path of the ultrasound wave. The wave starts from the transmit origin to the imaging point through the transmit focal point and propagates back to the 
receive element through the receive focal point. The length of TOF path is given by [7]:

$$
d_{t o f}=\left|\vec{r}_{v e}-\vec{r}_{e}\right| \pm\left|\vec{r}_{i p}-\vec{r}_{v e}\right| \pm\left|\vec{r}_{v e}-\vec{r}_{i p}\right|+\left|\vec{r}_{r}-\vec{r}_{v e}\right|,
$$

where, $\vec{r}_{v e}, \vec{r}_{e}, \vec{r}_{i p}, \vec{r}_{v e}$ are positions of the virtual element, the emitting element, the imaging point, and the receive element, respectively. The sign \pm in (1) represents whether the imaging point is in front or behind the virtual source. Thus, the delay time for looking up RF data $t_{d}$ is calculated as $t_{d}=d_{t o f} / c, c$ being the speed of sound.

The first stage RF lines are fed into the second beamformer. These RF lines contain the scatterer information within the focused beam, which is defined by an opening angle $\alpha$.

$$
\alpha=2 \arctan \frac{1}{2 F^{\#}} \text {. }
$$

The opening angle is determined by the $F^{\#}$, which is influenced by the fixed focal depth. Each image line from the first stage only carries the spatial information within the ultrasound beam. The signal outside the opening angle is discarded. The imaging point in the second stage is obtained by adding all the values from those RF data that intersect at that point (see Fig. 1).

\section{IIIIIIIIIIIIIIIIIIIIII}

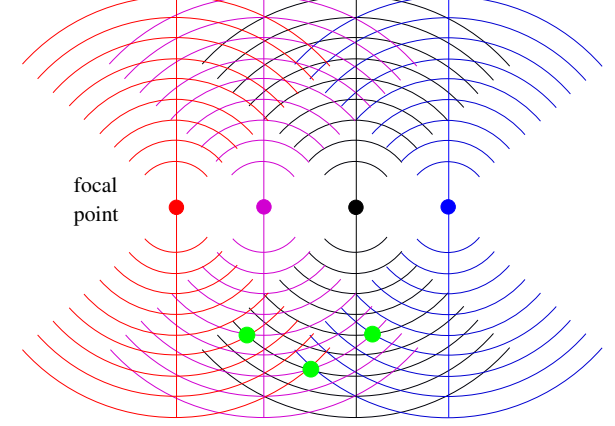

Fig. 1. Illustration of the second beamformer. Four image lines from the first stage are the input to the second stage here. They only carry limited information from the scatterer field which is indicated by waves. The high resolution points indicated as green dots are obtained by adding all low resolution points overlapping at that position.

Fig. 1 illustrates how the data are beamformed in the second beamformer. The opening angle defines the arc area that the ultrasound beam can cover and each high resolution point is composed of all low resolution points that can reach that position.

The HRI point at $\vec{r}_{i p}$ is constructed as

$$
\begin{gathered}
h\left(\vec{r}_{i p}\right)=\sum_{k=1}^{K(z)} \mathcal{W}\left(x_{k}, z\right) s_{k}\left(z_{k}\right) \\
z_{k}=\left|\vec{r}_{v e k}-\vec{r}_{e k}\right| \pm\left|\vec{r}_{i p}-\vec{r}_{v e k}\right| \pm\left|\vec{r}_{v e k}-\vec{r}_{i p}\right|+\left|\vec{r}_{e k}-\vec{r}_{v e k}\right|
\end{gathered}
$$

from (3) the HRI is formulated as the summation over the number of emissions $K$, the $x_{k}$ and $z_{k}$ are the lateral and axial positions which is define in (4). $\mathcal{W}$ is the apodization function, while $s_{k}$ denotes the spatial RF data from the first beamformer. All the LRIs contribute to a HRI. Therefore, a depth independent lateral resolution image can be achieved compared to conventional dynamic receive focusing imaging

\section{B. Flow velocity estimator}

This dual beamformer approach can be used for flow velocity estimation, because the HRI can be obtained continuously. At every emission the previous corresponding LRI is replaced by the latest LRI, thus, yielding a new HRI. As long as the replacement is repeated over the emission sequence, the HRI is available continuously. When scatterer are moving, every HRI is like a snapshot at every $T_{p r f}$ (pulse repetition time). As long as the shifted distance between two images can be estimated, the velocity can be obtained easily. This is the basic idea behind synthetic aperture flow estimation.

If the velocity is constant, the relationship between two HRIs can be expressed as [8]

$$
H^{n}(t)=H^{n-N}\left(t-2 \frac{\mathrm{v}_{z}}{c} N T_{p r f}\right),
$$

where $t$ is the propagation time from pulse emission, $n$ is the global emission index, $N$ is the number of emissions in one sequence, $v_{z}$ is the velocity along the axial direction and $T_{p r f}$ is the pulse repetition time. From (5) it is apparent that high resolution images obtained from different emission sequences are actually the time shifted version of each other. The time shift comes from the shifted position of moving scatterer, which makes the wave propagation path change, thus yielding a change in TOF. If this time shift can be estimated between two HRIs, the velocity can be calculated.

The time shift can be found by cross correlating two high resolution lines $H^{n}$ and $H^{n-N}$ and the velocity can be represented by the time shift. The cross correlation function can be formulated as [9]

$$
\begin{aligned}
R_{n-N, n}(\tau) & =\frac{1}{2 T} \int_{T} H^{(n-N)}(t) H^{(n)}(t+\tau) d t \\
& =\frac{1}{2 T} \int_{T} H^{(n-N)}(t) H^{(n-N)}\left(t-t_{s}+\tau\right) d t \\
& =R_{n-N, n-N}\left(\tau-t_{s}\right),
\end{aligned}
$$

where the $t_{s}$ gives the times shift of the peak of the cross correlation function. The velocity can be then expressed as

$$
\mathrm{v}_{z}=\frac{c t_{s}}{2 N T_{p r f}} .
$$

If the flow moves with a constant velocity, several cross correlation pairs $H^{n}$ and $H^{n-N}$ are subject to the same time shift and the maximum value of their cross correlation function is located at $\tau=t_{s}$ as well. Therefore, these cross correlation functions can be averaged to make the correct peak maximum and obtain better estimates. 
The above estimation is for a constant velocity, however, the blood velocity in the human vessel changes as a function of depth. In order to obtain the velocity profile along the depth, the RF data are divided into small segments. The above cross correlation function is calculated for each segments corresponding to the same depth. Velocities at the same depth are assumed to be almost the same so the value does not change significantly for several emissions. Then cross correlation functions from that sequence range can be averaged to improve the estimation.

Usually, signals are sampled with discrete time so the time shift $t_{s}$ can be represented as delay lag in the cross correlation function. Thus, the velocity can be reformulated as

$$
v_{z}=\frac{c k_{s}}{2 N f_{s} T_{p r f}},
$$

where $k_{s}$ is the delay lag in the cross correlation function and $f_{s}$ is the sampling frequency. A more precise lag can be found by interpolating the cross correlation function around the peak value [10]

$$
k_{\text {int }}=k_{s}-\frac{\hat{R}_{12}\left(k_{s}+1\right)-\hat{R}_{12}\left(k_{s}-1\right)}{2\left(\hat{R}_{12}\left(k_{s}+1\right)-2 \hat{R}_{12}\left(k_{s}\right)+\hat{R}_{1} 2\left(k_{s}-1\right)\right)} .
$$

Then the estimated velocity can be expressed as

$$
\hat{\mathrm{v}}_{z}=\frac{c k_{\text {int }}}{2 N f_{s} T_{\text {prf }}} .
$$

The important issue for cross correlation is that two high resolution images should be achieved from the same emission sequence. That maximizes the correlation function. The correlation level is low if two HRIs are not from exactly the same emission sequences. This can easily be done by applying recursive imaging. The HRI is available at every emission by replacing the corresponding LRI yielding the new cross correlation function. [3], [8]

\section{RESUlts}

The method is simulated by using Field II [11], [12]. The evaluation is quantified by the mean bias and standard deviation. The simulation parameters are shown in Table I.

\begin{tabular}{lll} 
Parameter & Value & Unit \\
\hline Central frequency & 7 & $\mathrm{MHz}$ \\
Sampling frequency & 120 & $\mathrm{MHz}$ \\
Speed of sound & 1540 & $\mathrm{~m} / \mathrm{s}$ \\
Number of elements & 192 & \\
Transducer pitch & 0.208 & $\mathrm{~mm}$ \\
Transducer height & 4.5 & $\mathrm{~mm}$ \\
Pulse repetition frequency & 5 & $\mathrm{KHz}$ \\
Transmit and receive focal depth (virtual source) & 10 & $\mathrm{~mm}$ \\
Number of transmit elements & 32 & \\
Number of receive elements & 64 & \\
\hline
\end{tabular}

TABLE I

PARAMETERS FOR FLOW SIMULATION.

The transducer has 192 elements with $\lambda$ pitch. The $F \#$ is determined by the length of the sub-aperture and the focal point. In the simulation it is fixed at $F \#=1.5$ as a starting point.

The scatterer positions in the flow vessel are simulated by a random uniform distribution and they have random Gaussian distributed amplitudes. A parabolic profile is used across the whole vessel. The vessel is $20 \mathrm{~mm}$ long with a diameter of $20 \mathrm{~mm}$ and the center of the vessel is $35 \mathrm{~mm}$ away from the transducer. The angle between the flow vessel and the axial beam is $45^{\circ}$.

The number of emissions is limited to $N=4$ in one emission sequence. The emission is repeated over the sequence, thus, the $n$ and $n+N$ sequence have the same sub-aperture to transmit and receive signals. Meanwhile, in the parameter study the spacing between emissions and number of averaged cross correlation functions are varied to see the robustness of the approach.

The simulation results are evaluated through the relative bias and relative standard deviation. The bias is defined as:

$$
v_{\text {bias }}=\frac{1}{N_{p} N_{v}} \sum_{i=1}^{N_{p}} \sum_{k=1}^{N_{v}} \hat{v}\left(\vec{r}_{k}, i\right)-v\left(\vec{r}_{k}\right)
$$

where $N_{p}$ is the number of estimated profiles, $N_{v}$ is the number of samples in one profile, $\vec{r}_{k}$ represents the position for velocity estimation, $\hat{v}\left(\vec{r}_{k}, i\right)$ is the estimated velocity at $\vec{r}_{k}$ in $i_{t h}$ profile, and $v\left(\vec{r}_{k}\right)$ is the true velocity profile. The standard deviation is defined as:

$$
v_{s t d}=\sqrt{\frac{1}{N_{p} N_{\mathrm{v}}} \sum_{i=1}^{N_{p}} \sum_{k=1}^{N_{\mathrm{v}}}\left(\hat{\mathrm{v}}\left(\vec{r}_{k}, i\right)-\overline{\mathrm{v}}\left(\vec{r}_{k}\right)\right)^{2}},
$$

where $\bar{v}\left(\vec{r}_{k}\right)$ is the mean velocity profile estimate. The relative bias and standard deviation are relative to the peak velocity in the vessel.

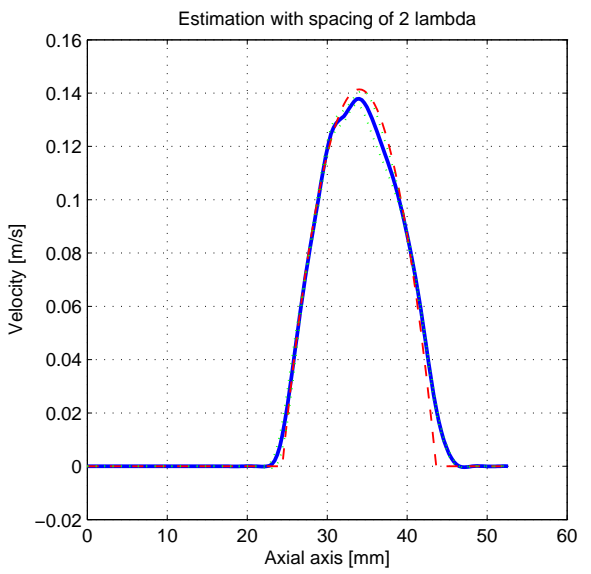

Fig. 2. Velocity profiles at the center. Blue solid line is the estimated profile, red dashed line is the true profile, and green dot lines are \pm three standard deviations.

Fig. 2 shows the result of the new estimator and the estimated velocity profiles at the center are shown in the figure. Blue solid line gives the mean of 64 estimates. The 

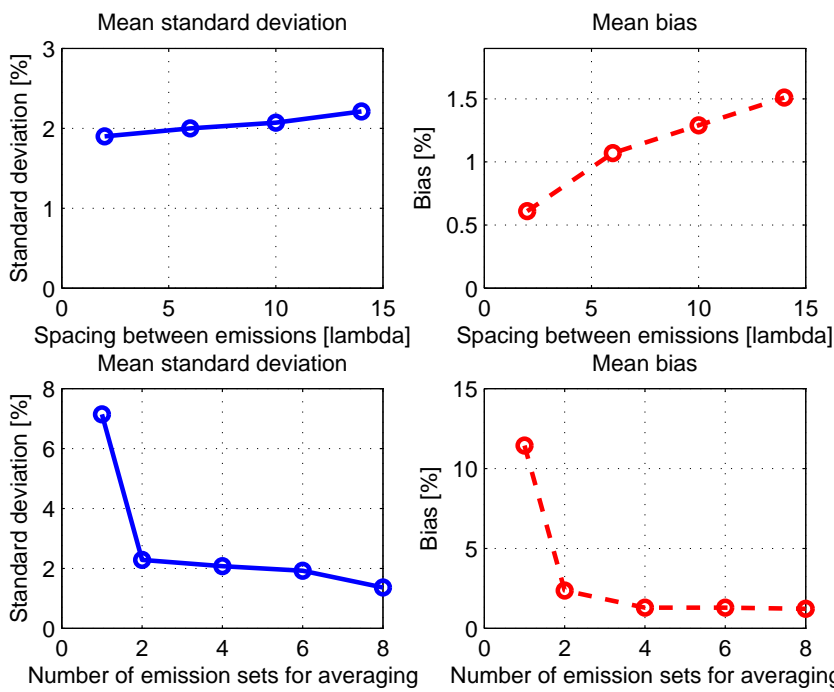

Fig. 3. The statistics for the performance as a function of spacing and averaging number. The top row shows standard deviation and bias as a function of spacing. The second row shows the performance as a function of the number of emission sets used for averaging.

larger spacing is supposed to cover a wider area, but a larger area is obtained at the expense of a slight increase in standard deviation. The parameter study gives the performance of varying the spacing from $2 \lambda$ to $14 \lambda$. When four emissions are further apart, the standard deviation at the center only changes slightly.

The quantitative statistics are shown in Fig. 3. The blue solid line represents the mean standard deviation, while the red dashed line is the mean bias. The first row illustrates the influence of spacing between beams. Although the performance is improved with smaller spacing, the magnitude is small, meaning it has limited influence on the performance.

The second row illustrates how averaging affect performance. A high number of emission sets for averaging yields improved estimations. Four emission sets are used for averaging as no major improvement is seen beyond that. Therefore, the previous simulations are done by choosing four emission sequences to average cross correlation functions.

Fig. 4 provides an overview of the performance over the lateral position. Each curve represents the root mean square error as a function of the lateral position with a certain spacing. The estimation accuracy decreases when the vessel is further away from the center and increased spacing decreases the accuracy.

\section{CONCLUSION}

A new method for flow estimation is presented in this paper. A two-stage beamformation is applied for imaging, where the first stage beamformer creates RF lines and the second stage beamformer creates the image with full dynamic focusing. The velocity can be obtained by cross correlating the corresponding image lines. The approach is verified by Field II. The performance of the estimation is affected by the spacing

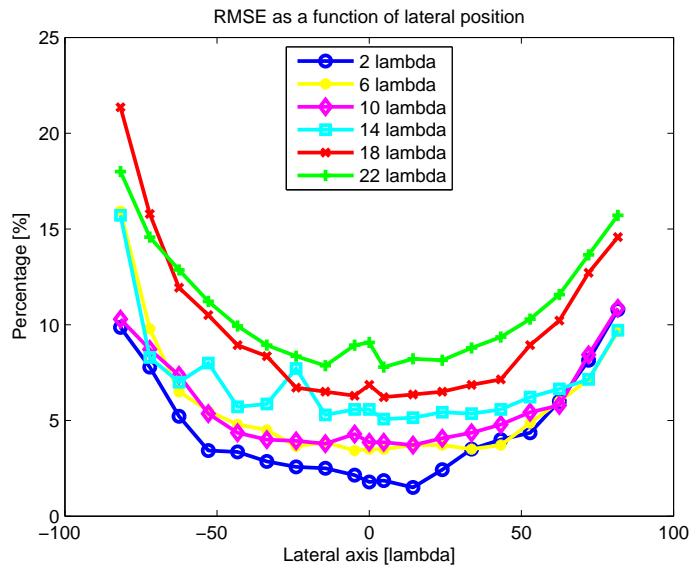

Fig. 4. The root mean square error of the performance at different lateral position with varied interspace.

between emissions and the number of sets for averaging. The approach has its limitation in covering a large area, and this is the topic for further investigation.

\section{REFERENCES}

[1] J. A. Jensen and N. Oddershede, "Estimation of velocity vectors in synthetic aperture ultrasound imaging.," IEEE Trans. Med. Imag., pp. 16371644, 2006.

[2] M. O'Donnell and L. J. Thomas, "Efficient synthetic aperture imaging from a circular aperture with possible application to catheter-based imaging," IEEE Trans. Ultrason., Ferroelec., Freq. Contr., vol. 39, pp. 366-380, 1992.

[3] S. I. Nikolov, K. Gammelmark, and J. A. Jensen, "Recursive ultrasound imaging," in Proc. IEEE Ultrason. Symp., vol. 2, pp. 1621-1625, 1999.

[4] J. A. Jensen, S. Nikolov, K. L. Gammelmark, and M. H. Pedersen, "Synthetic aperture ultrasound imaging," Ultrasonics, vol. 44, pp. e5e15, 2006.

[5] C. H. Frazier and W. D. O'Brien, "Synthetic aperture techniques with a virtual source element," IEEE Trans. Ultrason., Ferroelec., Freq. Contr., vol. 45, pp. 196-207, 1998.

[6] S. I. Nikolov and J. A. Jensen, "Virtual ultrasound sources in highresolution ultrasound imaging," in Proc. SPIE - Progress in biomedical optics and imaging, vol. 3, pp. 395-405, 2002.

[7] J. Kortbek and J. A. Jensen, "Synthetic aperture sequential beamforming," in Proc. IEEE Ultrason. Symp., pp. 966-969, 2008.

[8] S. I. Nikolov and J. A. Jensen, "Velocity estimation using synthetic aperture imaging," in Proc. IEEE Ultrason. Symp., pp. 1409-1412, 2001.

[9] M. H. Bae and M. K. Jeong, "A study of synthetic-aperture imaging with virtual source elements in B-mode ultrasound imaging systems," in IEEE Trans. Ultrason., Ferroelec., Freq. Contr., vol. 47, pp. 15101519, 2000.

[10] S. G. Foster, A pulsed ultrasonic flowmeter employing time domain methods. PhD thesis, Dept. Elec. Eng., University of Illinois, Urbana, Ill., 1985.

[11] J. A. Jensen, "Field: A program for simulating ultrasound systems," Med. Biol. Eng. Comp., vol. 10th Nordic-Baltic Conference on Biomedical Imaging, Vol. 4, Supplement 1, Part 1, pp. 351-353, 1996 b.

[12] J. A. Jensen and N. B. Svendsen, "Calculation of pressure fields from arbitrarily shaped, apodized, and excited ultrasound transducers," IEEE Trans. Ultrason., Ferroelec., Freq. Contr., vol. 39, pp. 262-267, 1992. 